\title{
Expression and the clinical significance of hPTTG1 in gastric cancer
}

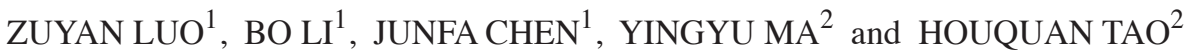 \\ ${ }^{1}$ Department of Radiology, Zhejiang Provincial People's Hospital; \\ ${ }^{2}$ Key Laboratory of Gastroenterology of Zhejiang Province, Hangzhou 310014, P.R. China
}

Received April 4, 2012; Accepted July 3, 2012

DOI: $10.3892 / \mathrm{mmr} .2012 .1161$

\begin{abstract}
The aim of this study was to investigate the expression and clinical significance of hPTTG1 in gastric cancer. Immunohistochemistry was performed to determine the expression of hPTTG1 in gastric cancer tissues. Results showed that the positive expression of hPTTG1 in gastric cancer tissues was $60.00 \%$, while in adjacent normal tissues it was $17.78 \%$. The expression of hPTTG1 was correlated with differentiation levels, clinical classification and lymph node metastasis, but did not correlate with gender, age or pathological types. hPTTG1 was, therefore, overexpressed in gastric cancer tissues. The progression of gastric cancer was found to be correlated with the upregulation of the expression of hPTTG1. hPTTG1 detection may be helpful in evaluating the ability of the clinical classification and lymph node metastasis in gastric cancer to predict outcomes. These factors act as indicators of the biological behavior of gastric cancer and are fairly good markers for prognosis and therapy.
\end{abstract}

\section{Introduction}

Pituitary tumor-transforming gene (PTTG) was first identified in rat pituitary tumor by differential display PCR (1). At present, three members of the PTTG family have been identified in humans (2). Among those, the hPTTG1 has a human securin function, while playing an important role in tumorigenesis and tumor metastasis $(3,4)$. The hPTTG1 was found to be overexpressed in a variety of cancers, such as lung cancer, thyroid tumor, as well as ovarian and esophageal cancer (5-8). Normally, the hPTTG1 expression can only be detected in testis, thymus, embryo and liver (4). The abnormal expressions of hPTTG1 implied tumorigenesis or tumor metastasis. However, the correlation between the expression of hPTTG1 and gastric cancer has yet to be clarified.

Correspondence to: Dr Bo Li, Department of Radiology, Zhejiang Provincial People's Hospital, 158 Shangtang Rd, Hangzhou 310014, P.R. China

E-mail: zuyanluo@126.com

Key words: human pituitary tumor transforming gene 1, gastric cancer, immunohistochemistry
Cancer has been the leading cause of mortality in many countries $(9,10)$, with gastric cancer being the second leading cause of cancer-related mortality in both males and females worldwide (11). Gastric cancer is still widespread in China, as seen in a statistical report demonstrating that China accounts for approximately $50 \%$ of the global gastric cancer burden (11). It is widely accepted that early discovery indicates a good prognosis in cancer therapy. Thus, it is urgent to develop a facilitating technique to detect tumors, at least tumor metastasis.

The hPTTG1 gene has transcriptional activity and securin functions (12). Overexpression of the hPTTG1 promotes lymph node metastasis in human esophageal carcinoma (8). Findings of previous studies revealed that the downregulation of the expression of hPTTG1 resulted in the suppression of tumor growth $(13,14)$. We hypothesized that the abnormal overexpression of hPTTG1 was involved in tumorigenesis and tumor metastases in gastric cancer.

Lymph node metastasis is an important prognostic factor in early gastric cancer, which indicates the risk of recurrence (15). Thus it is necessary to detect whether lymph node metastasis is present prior to curative resection. The removal of lymph nodes should be carried out during surgery. Therefore, a reliable method is needed to accurately detect lymph node metastasis in primary gastric cancer. However, the expression and correlation of hPTTG1 with clinicopathological parameters in gastric cancer has not been reported yet. Therefore, SP immunohistochemical technology was applied to investigate the expression of hPTTG1 in gastric cancer and adjacent normal tissues and explore its correlation with clinicopathological features and significance.

The aim of the present study was to develop a facilitation method to detect gastric cancer or lymph node metastasis in gastric cancer. We found that the expression of hPTTG1 was associated with gastric cancer and lymph node metastasis in gastric cancer. We suggested that the immunohistochemistry staining of hPTTG1 may be used as an effective tool to reveal its clinicopathological parameters and significance in gastric cancer.

\section{Materials and methods}

Patients and tissues. Gastric cancer and adjacent normal tissues were collected at the time of surgery from patients with 
Table I. Relationship between hPTTG1 expression and types of tissue.

\begin{tabular}{|c|c|c|c|c|c|c|c|c|}
\hline Types of tissue & $\mathrm{n}$ & -- & + & ++ & +++ & Positive rate $(\%)$ & $\chi^{2}$ & P-value \\
\hline Gastric cancer & 45 & 18 & 8 & 8 & 11 & 60.00 & 16.875 & 0.000 \\
\hline Adjacent normal tissues & 45 & 37 & 1 & 3 & 4 & 17.78 & & \\
\hline
\end{tabular}

gastric cancer between May, 2004 and May, 2008. The tumor samples were confirmed by histopathological examination. Prior to surgery, the patients did not receive radiation treatment, chemo-treatment or biological therapy for the tumor. The adjacent normal tissues were obtained from a site $5 \mathrm{~cm}$ away from the tumor and confirmed histologically. These samples were obtained from 28 males and 17 females. The average age was 59.5 years (range, $45-79$ ). The tumor-node-metastasis (TNM) stages were classified according to the Union for International Cancer Control (UICC) 1987 of TNM classification. Five cases of stage I, 10 cases of stage II, 20 cases of stage III and 10 cases of stage IV were involved in our study. Of the 45 cases, 31 had a lymph node metastasis, and 14 cases were not detected at surgery. In the present study, there were 16 cases with welldifferentiated, 15 with moderately-differentiated and 14 cases with poorly-differentiated adenocarcinoma.

Immunohistochemistry. An SP immunohistochemical staining kit was purchased from Beijing Zhongshan Bio-tech Co., Ltd. Immunohistochemistry was performed according to the manufacturer's instructions. Briefly, gastric cancer tissues and adjacent normal tissues were dissected out, followed by fixing in Bouin's solution for $24 \mathrm{~h}$. After an extensive wash in $70 \%$ ethanol, the samples were dehydrated and embedded in paraffin. The paraffin blocks were cut at $5 \mu \mathrm{m}$. The deparaffinized sections were incubated with a $3 \%$ hydrogen peroxide solution for $30 \mathrm{~min}$. After being washed with PBS solution twice, the slides with sample sections were incubated with $0.01 \mathrm{M}$ citrate solution at $95^{\circ} \mathrm{C}$ for $10 \mathrm{~min}$ to retrieve the epitope. After cooling to room temperature, the glass slides were rinsed with PBS solution twice. The slides were incubated with NGS-PBS (PBS containing 10\% normal goat serum) for $30 \mathrm{~min}$, and then washed with PBS solution.

Primary antibody, rabbit anti-human PTTG1 (Santa Cruz Biotechnology Inc., Santa Cruz, CA, USA) at a dilution of 1:100 was dropped onto the slides. After incubation at $37^{\circ} \mathrm{C}$ for $1 \mathrm{~h}$ the slides were washed with PBS solution twice. Subsequently, the secondary antibody (biotin-conjugated goat anti-rabbit IgG, Beijing Zhongshan Bio-tech Co., Ltd., China) at a dilution of $1: 100$ was dropped onto the slides and incubated at $37^{\circ} \mathrm{C}$ for $30 \mathrm{~min}$. After washing with PBS solution twice, the slides were applied to 3,3'-diaminobenzidine (Dako Tech Co., Ltd, Denmark) staining. The positive slides were defined according to a previous study (16). Briefly, the slides were defined as i) (+) if from $10-25 \%$ of highest possible frequency (HPF), showing a positive signal with a magnification of $x 400$; ii) (++) if from $25-50 \%$ of HPF, showing positive signal with magnification at $\mathrm{x} 400$ and iii) $(+++)$ if $>50 \%$ of HPF, showing positive signal with a magnification of $x 400$. Each slide was examined by 2 pathologists, respectively. In case of a disagreement, the slide was determined by a third pathologist.

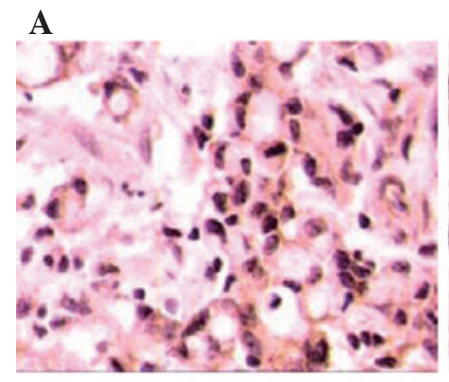

B

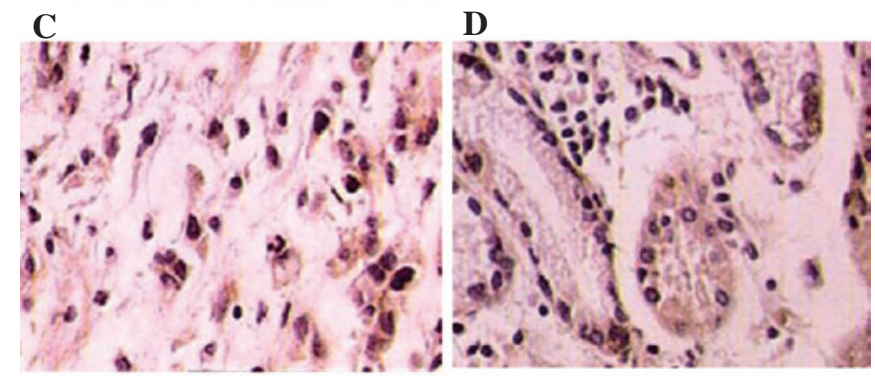

Figure 1. Immunohistochemical analysis of the expression of hPTTG1 in gastric cancer and adjacent normal tissues. Representative images are shown as indicated. (A) Expression of hPTTG1 in signet-ring cell carcinoma, undifferentiated carcinoma with positive staining. (B) hPTTG1 in moderately differentiated adenocarcinoma with positive staining. (C) hPTTG1 in poorly differentiated adenocarcinoma with positive staining. (D) hPTTG1 in adjacent normal tissues with positive staining. Magnification, $\mathrm{x} 400$.

Statistical analyses. Data were presented as the mean \pm SD. Statistical analysis was performed using SPSS 11.0.0 (SPSS Inc., Chicago). Correlations were evaluated using the Chi-square test. $\mathrm{p}<0.05$ was considered to indicate a statistically significant difference.

\section{Results}

Expression of hPTTG1 in gastric cancer and the adjacent normal tissues. We detected the expression of hPTTG1 in different cell types and tissues (Fig. 1). A number of nuclei exhibited dark staining in signet-ring cell carcinoma, poorly differentiated adenocarcinoma and adjacent normal tissues, possibly indicating a poor prognosis. The statistical analysis revealed that the expression of hPTTG1 was associated with gastric cancer as compared to the adjacent normal tissues (Table I).

Correlation between hPTTG1 expression and the clinicopathological variables of gastric cancer. The statistical analysis demonstrated that the expression of hPTTG1 was associated with differentiation levels, clinical classification and lymph node metastasis in gastric cancer. This finding suggests that the overexpression of hPTTG1 may indicate poor prognosis. 
Table II. Relationship between hPTTG1 expression and clinicopathologic parameters in gastric cancer.

\begin{tabular}{|c|c|c|c|c|c|c|c|c|}
\hline Clinicopathologic variables & $\mathrm{n}$ & -- & + & ++ & +++ & Positive rate $(\%)$ & $\chi^{2}$ & P-value \\
\hline \multicolumn{9}{|l|}{ Gender } \\
\hline Male & 28 & 12 & 4 & 4 & 8 & 57.14 & \multirow[t]{2}{*}{1.079} & \multirow[t]{2}{*}{0.299} \\
\hline Female & 17 & 10 & 3 & 1 & 3 & 41.18 & & \\
\hline \multicolumn{9}{|l|}{ Age, years } \\
\hline$\geq 60$ & 33 & 13 & 5 & 4 & 11 & 60.61 & \multirow[t]{2}{*}{0.019} & \multirow[t]{2}{*}{0.891} \\
\hline$<60$ & 12 & 5 & 2 & 3 & 2 & 58.33 & & \\
\hline \multicolumn{9}{|l|}{ Pathological types } \\
\hline Adenocarcinoma & 34 & 14 & 4 & 7 & 9 & 58.82 & \multirow[t]{3}{*}{0.311} & \multirow[t]{3}{*}{0.856} \\
\hline Mucinous adenocarcinoma & 8 & 4 & 0 & 2 & 2 & 50.00 & & \\
\hline Signet-ring cell carcinoma & 3 & 1 & 0 & 2 & 0 & 66.67 & & \\
\hline \multicolumn{9}{|l|}{ Differentiation levels } \\
\hline High & 16 & 13 & 2 & 1 & 0 & 18.75 & \multirow[t]{3}{*}{13.582} & \multirow[t]{3}{*}{0.001} \\
\hline Moderate & 15 & 7 & 4 & 3 & 2 & 60.00 & & \\
\hline Poor & 14 & 2 & 3 & 1 & 8 & 85.71 & & \\
\hline \multicolumn{9}{|l|}{ Clinical classification } \\
\hline TI+TII & 15 & 10 & 3 & 2 & 0 & 33.33 & \multirow[t]{2}{*}{6.667} & \multirow[t]{2}{*}{0.010} \\
\hline TIII+TIV & 30 & 8 & 5 & 7 & 10 & 73.33 & & \\
\hline \multicolumn{9}{|l|}{ Lymph node metastasis } \\
\hline Presence & 31 & 8 & 5 & 6 & 12 & 74.19 & \multirow[t]{2}{*}{6.075} & \multirow[t]{2}{*}{0.014} \\
\hline Absence & 14 & 9 & 3 & 2 & 0 & 35.71 & & \\
\hline
\end{tabular}

Moreover, we found that the expression of hPTTG1 did not correlate with gender, age or pathological types (Table II).

\section{Discussion}

The abnormal expression of hPTTG1 is involved in tumorigenesis and tumor metastasis (17). Our study showed that the immunohistochemical analysis of hPTTG1 was suitable for detecting different cell types and tissues in gastric cancer. We also showed that the expression of hPTTG1 is correlated with gastric cancer as compared to the adjacent normal tissues. This finding indicated that the expression of hPTTG1 may be a useful reference to gastric cancer detection.

Results from our study demonstrated that the expression of hPTTG1 was associated with differentiation levels, clinical classification and lymph node metastasis in primary gastric cancer. Poorly differentiated cancer and higher clinical classification indicated poor prognosis. Thus, this finding suggests that the expression of hPTTG1 is associated with poor prognosis. Lymph node metastasis is the most important prognostic factor for patients with early gastric cancer. Since the presence of lymph node metastasis indicates a poor prognosis on patients with early gastric cancer, it is suggested that the first and second tier lymph nodes be removed during surgery (15). The removal of lymph nodes was reported to be associated with an extended period of survival (18). Therefore, the detection of lymph node metastasis is valuable for early gastric cancer patients. We suggest that the expression of hPTTG1 be considered in early gastric cancer therapy.

A statistical report of 2008 showed that age-standardized incidence rates are approximately twice as high in men as in women (11). The expression of hPTTG1, however, did not correlate with gender. Moreover, we found that the expression of hPTTG1 was also not associated with age or pathological types.

Our results describe hPTTG1 expression as a potential tool for the assessment of tumor aggressiveness. The immunohistochemistry of hPTTG1 expression may be a convenient way to large-scale analyses or routine pathological diagnosis. This tool may have special value for early gastric cancer patients, for the detection of lymph node metastasis and extention of their period of survival.

\section{Acknowledgements}

This study was supported by a grant from the National Special Funds for the Health Research Foundation of China (no. 200802112) and the National Natural Science Foundation of China (no. 81071991).

\section{References}

1. Pei L and Melmed S: Isolation and characterization of a pituitary tumor-transforming gene (PTTG). Mol Endocrinol 11: 433-441, 1997.

2. Zhang X, Horwitz GA, Prezant TR, Valentini A, Nakashima M, Bronstein MD and Melmed S: Structure, expression, and function of human pituitary tumor-transforming gene (PTTG). Mol Endocrinol 13: 156-166, 1999.

3. Chen L, Puri R, Lefkowitz EJ and Kakar SS: Identification of the human pituitary tumor transforming gene (hPTTG) family: molecular structure, expression, and chromosomal localization. Gene 248: 41-50, 2000.

4. Hamid T and Kakar SS: PTTG and cancer. Histol Histopathol 18: 245-251, 2003. 
5. Shah PP, Fong MY and Kakar SS: PTTG induces EMT through integrin alpha(V)beta(3)-focal adhesion kinase signaling in lung cancer cells. Oncogene: Nov. 14, 2011 (E-pub ahead of print).

6. Chintharlapalli S, Papineni S, Lee SO, Lei P, Jin UH, Sherman SI, Santarpia L and Safe S: Inhibition of pituitary tumor-transforming gene-1 in thyroid cancer cells by drugs that decrease specificity proteins. Mol Carcinogen 50: 655-667, 2011.

7. El-Naggar SM, Malik MT and Kakar SS: Small interfering RNA against PTTG: A novel therapy for ovarian cancer. Int J Oncol 31: 137-143, 2007.

8. Yan S, Zhou CQ, Lou XM, Xiao ZF, Zhu HX, Wang QF, Wang YH, Lu N, He S, Zhan QM, et al: PTTG overexpression promotes lymph node metastasis in human esophageal squamous cell carcinoma. Cancer Res 69: 3283-3290, 2009.

9. Jung KW, Park S, Kong HJ, Won YJ, Boo YK, Shin HR, Park EC and Lee JS: Cancer Statistics in Korea: incidence, mortality and survival in 2006-2007. J Korean Med Sci 25: 1113-1121, 2010.

10. Boyle P and Ferlay J: Cancer incidence and mortality in Europe, 2004. Ann Oncol 16: 481-488, 2005.

11. Ferlay J, Shin HR, Bray F, Forman D, Mathers C and Parkin DM: Estimates of worldwide burden of cancer in 2008: GLOBOCAN 2008. Int J Cancer 127: 2893-2917, 2010.
12. Tong Y and Eigler T: Transcriptional targets for pituitary tumortransforming gene-1. J Mol Endocrinol 43: 179-185, 2009.

13. Panguluri SK, Yeakel C and Kakar SS: PTTG: an important target gene for ovarian cancer therapy. J Ovarian Res 1: 6, 2008.

14. Kakar SS and Malik MT: Suppression of lung cancer with siRNA targeting PTTG. Int J Oncol 29: 387-395, 2006.

15. Okamura T, Tsujitani S, Korenaga D, Haraguchi M, Baba H, Hiramoto Y and Sugimachi K: Lymphadenectomy for cure in patients with early gastric cancer and lymph node metastasis. Am J Surg 155: 476-480, 1988.

16. Lu CD, Altieri DC and Tanigawa N: Expression of a novel antiapoptosis gene, survivin, correlated with tumor cell apoptosis and p53 accumulation in gastric carcinomas. Cancer Res 58: 1808-1812, 1998

17. Salehi F, Kovacs K, Scheithauer BW, Lloyd RV and Cusimano M: Pituitary tumor-transforming gene in endocrine and other neoplasms: a review and update. Endocr Relat Cancer 15: 721-743, 2008.

18. Sano T, Sasako M, Kinoshita T and Maruyama K: Recurrence of early gastric cancer. Follow-up of 1475 patients and review of the Japanese literature. Cancer 72: 3174-3178, 1993. 